Open Access Full Text Article

\title{
Low-level versus high-level placement of gold plates in the upper eyelid in patients with facial palsy
}

This article was published in the following Dove Press journal:

Clinical Ophthalmology

29 June 2011

Number of times this article has been viewed

\author{
Tarek A Amer' \\ Hisham M El-Minawi' \\ Malak I El-Shazly² \\ 'Department of Plastic Surgery, \\ 2Department of Ophthalmology, Cairo \\ University, Cairo, Egypt
}

Background: Lagophthalmos is a condition that results from facial paralysis causing functional as well as esthetic problems. This condition can be treated by a range of techniques, including tarsorrhaphy, facial slings, and canthopexies. Gold plates provide a solution for temporary or permanent lagophthalmos resulting from facial paralysis. This study discusses the use of gold plates in the treatment of lagophthalmos but with the introduction of gold plates in two different positions in the upper lids.

Methods: Group 1 (38 eyes) had a low level of placement ( $2 \mathrm{~mm}$ from the lid margin) of gold plates, while Group 2 (23 eyes) had a high level of placement ( $5 \mathrm{~mm}$ from the lid margin).

Results: Noticeable bulge was seen in $18.4 \%$ of Group 1 eyes compared with $13 \%$ in Group 2, and migration of the plate occurred in $2.6 \%$ and $0 \%$ of eyes in Group 1 and Group 2, respectively, as well as ptosis $(7.8 \%$ and $4.3 \%)$ and conjunctival perforation ( $0 \%$ and $4.3 \%)$. The degree of improvement of eyelid closure, keratopathy, and visual acuity were the same for both techniques.

Conclusion: Placement of gold plates at a higher level could avoid some of the drawbacks of lower level placement of these plates, such as upper eyelid bulge and ptosis, especially given the thinning of the eyelids and orbicularis muscles that occurs in facial palsy.

Keywords: lagophthalmos, tarsorrhaphy, gold plates, keratopathy

\section{Introduction}

Lagophthalmos, which is the inability to close the eyelids completely, is a serious condition caused by orbicularis oculi muscle paresis or paralysis. The orbicularis oculi muscle is innervated by the facial nerve. Paresis of the orbicularis oculi muscle leads to diminished blinking, lagophthalmos, and impairment of the nasolacrimal pump. With diminished orbicularis tone, the lower lid becomes lax, and the resting position of the lower eyelid drops. Corneal surface disruption results from both increased surface exposure and from the disruption of tear film caused by inadequate blinking mechanisms. Alteration in VIIth nerve function may also affect the secretion of tears and result in an absent Bell's phenomenon. Associated loss of Vth nerve function further increases the risk of surface disruption caused by loss of the protective mechanism of corneal sensation and by loss of its protective neurotropic effect on growth of the corneal epithelium. ${ }^{1}$ The resulting corneal problems include epithelial defects, stromal thinning, exposure keratitis, bacterial infection, perforation, and blindness. Lagophthalmos may be the result of the residual effect of VIIth cranial nerve damage, which can be congenital (moebius syndrome), acquired (Bell's palsy, vascular lesions), or iatrogenic (during surgery), or arise from trauma, infection, or degenerative diseases. ${ }^{2,3}$
Correspondence: Malak Ismail El-Shazly Department of Ophthalmology, Cairo University, 5 Ebn El Nabieh Street,

Zamalek, Cairo, Egypt

Tel +20122244843

Email melshazly75@hotmail.com 
Treatment of lagophthalmos may be conservative and symptomatic, such as eye ointments, drops, lubricants, taping and moisture chambers, soft contact lenses, and scleral shells, with or without the need for surgical intervention. Surgical intervention may be required in patients who have failed medical therapy or in whom facial paralysis is not expected to improve. ${ }^{4}$ Surgical techniques include simple tarsorrhaphy, which may be temporal, medial, or both, ${ }^{5}$ canthoplasty, palpebral springs, silicone slings, eyelid magnets, temporalis muscle transfers, and nerve grafts. These methods can result in narrowing of the lid aperture, increase the risk of extrusion, and have the disadvantage of a very high rate of mechanical failure.

Another widely used surgical technique is the implantation of a gold weight. A gold weight placed on the upper eyelid on the affected side enables eyelid closure by gravity, with relaxation of the levator muscle of the upper eyelid. It is considered to be a physiological method which does not interfere with esthetic eyelid opening and preserves the field of vision. Another advantage is that the procedure can be reversed without changing eyelid anatomy. ${ }^{5,6}$

Several recent modifications to the implant as well as the surgical technique have been reported to minimize complications. ${ }^{7-10}$ In this study, we evaluated the different ocular results and complications, as well as esthetic satisfaction, in patients who underwent gold weight implantation $2 \mathrm{~mm}$ or $5 \mathrm{~mm}$ from the lid margin.

\section{Methods and materials}

Sixty-one eyes of 60 patients ( 32 male and 28 female) were enrolled in this study. The mean age of the patients was 34 (25-59) years. Sixty-one gold plates were used to treat lagophthalmos in 60 patients with facial nerve paralysis (one patient had moebius syndrome with bilateral complete facial nerve paralysis). Other etiologies included congenital facial nerve paralysis, cerebellopontine angle tumor excision, Bell's palsy, fractured base of the skull, and iatrogenic facial nerve injury. Etiologies of the facial paralysis are shown in Table 1.

A thorough preoperative history and informed consent were obtained. In addition, a series of preoperative and postoperative

Table I Etiologies of facial paralysis

\begin{tabular}{ll}
\hline Etiology & Eyes \\
\hline Tumor excision & $26(42.62 \%)$ \\
Fracture base & $10(16.39 \%)$ \\
latrogenic & $5(8.19 \%)$ \\
Bell's palsy & $14(22.95 \%)$ \\
Congenital & $6(9.83 \%)$ \\
\hline
\end{tabular}

measurements of the ipsilateral and contralateral eyelids was performed. These included palpebral fissure height (distance between eyelid margins in primary gaze), lagophthalmos (distance between eyelid margins with gentle closure), inferior scleral show (amount of sclera visible beneath the lower limbus with gentle closure, MRD1 [marginal "superior" reflex distance 1 , ie, distance between upper eyelid margin and corneal light reflex in primary gaze] and MRD2 [marginal "inferior" reflex distance 2, ie, distance between lower eyelid margin and corneal light reflex in primary gaze]). Visual acuity was recorded, and examination of the cornea and ophthalmic sensation was done preoperatively and postoperatively. Different ocular symptoms, eg, burning and tearing, and the need for utilization of artificial tears were noted. Preoperative and postoperative photographs were taken, and the patients were followed up monthly for at least one year. At the start of the study, we placed the gold plates at a low level of insertion (first technique, $n=38$ eyes), but during the course of the study, we observed an increased incidence of noticeable bulge and ptosis, so we shifted to placing the gold plate at a higher level to overcome these two problems (second technique, $n=23$ eyes).

\section{Gold plate}

The custom-made weights were made of 24-carat gold, and were $16-18 \mathrm{~mm}$ long, $5 \mathrm{~mm}$ in height, and fashioned as a rectangle with round borders. The body of the lid load had three to five holes to facilitate suspension (Figure 1). Each gold weight had a smooth surface and weighed $0.9-1.5 \mathrm{~g}$. The correct weight was selected preoperatively by taping different weights to the upper eyelid. The correct weight should close the eye on levator relaxation and not cause ptosis on levator contraction. Intraoperatively, the malleable gold plate was shaped with convexity on its anterior surface and concavity on its posterior surface, which allowed the gold weight inside the eyelid to rest smoothly along the globe.

\section{Surgical techniques}

Both techniques were performed in the same fashion with one exception, ie, the distance of the pocket from the
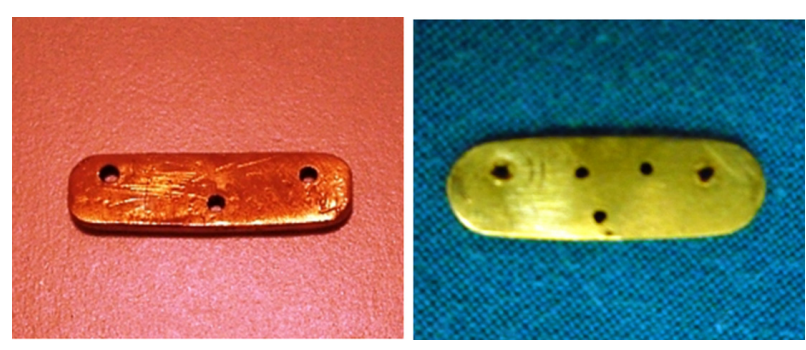

Figure I Gold plates with three and five holes to facilitate suspension. 
lid margin. The surgical procedure was done either with local infiltrative anesthesia and intravenous sedation or under general anesthesia. An incision about $1 \mathrm{~cm}$ long was made in the skin crease in the upper eyelid centered over the pupil. The orbicularis oculi was then slit open down to the tarsal plate and a pocket was created beneath the muscle over the middle third of the upper eyelid.

The orbital septum and the levator aponeurosis were always posterior to the gold plate in both techniques, and care was taken to avoid injury to either of these structures. Using the first technique, the lower edge of the pocket was kept at a distance of $2 \mathrm{~mm}$ from the lid margin, and using the second technique it was kept at a distance of $5 \mathrm{~mm}$ from the lid margin. The gold plate was inserted into the pocket and fixed in the tarsal plate through three holes with 6-0 Prolene. The decision to make extra holes in the gold plate was made intraoperatively to achieve the most convenient holes for fixation. The orbicularis oculi was closed using 6-0 Vicryl and the skin was closed with 7-0 Prolene. An eye dressing was used for 24 hours and the patient was discharged with oral and topical antibiotics on the first postoperative day. Figures 2 and 3 show patients with gold plates inserted using the first and second techniques, respectively.

\section{Results}

Thirty-eight eyes (62.3\%) underwent gold plate placement at a low level ( $2 \mathrm{~mm}$ from the eyelid margin, first technique) and 23 eyes (37.7\%) underwent gold plate placement at a high level ( $5 \mathrm{~mm}$ from the lid margin, second technique). One patient had moebius syndrome with complete bilateral facial nerve paralysis. Average time from the onset of facial paralysis to the lid loading procedure was 2.5 years (range 20 days to 5 years). The most commonly weight used with the first technique was $1.1 \mathrm{~g}$ in 14 eyes followed by $1.2 \mathrm{~g}$ in another five eyes. The most commonly used weight in the second technique was $1.2 \mathrm{~g}$ in seven eyes followed by $1.3 \mathrm{~g}$

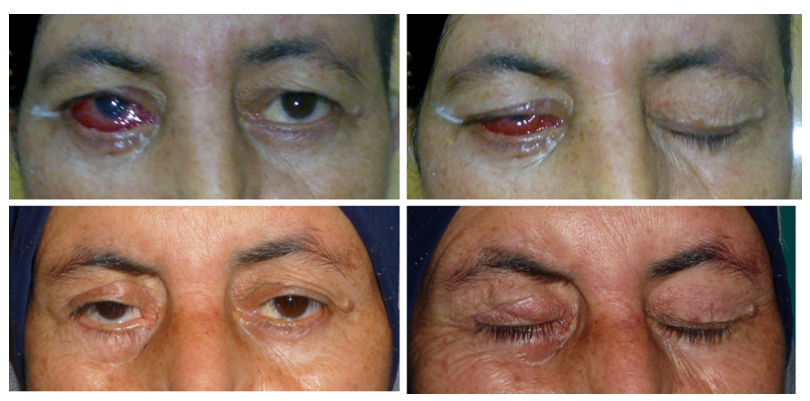

Figure 2 Patient with low level of gold plate insertion, preoperatively with eye open (above left), preoperatively with eye closed (above right), postoperatively with eye open (below left), and postoperatively with eye closed (below right).

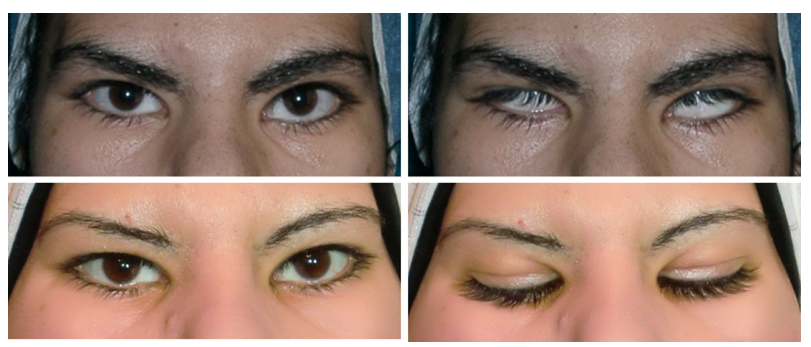

Figure 3 Patient with high level of insertion of gold plate, preoperatively with eye open (above left), preoperatively with eye closed (above right), postoperatively with eye open (below left), and postoperatively with eye closed (below right).

in another six eyes. The gold weights used in all other eyes weighed $0.9-1.5 \mathrm{~g}$.

Exposure keratitis was graded preoperatively and postoperatively on a scale of $0-4$. No keratopathy was rated 0 , and mild superficial punctate keratopathy anywhere over the cornea was rated 1 . If the superficial punctate keratopathy was located in the inferior quarter of the cornea, the rating was 2 , in the inferior one-third to one-half of the cornea, the rating was 3 , and if it covered more than half of the cornea or if the erosion existed anywhere over the cornea, the rating was 4 . For the first technique, the average preoperative and postoperative ratings were 1.40 and 0.40 , respectively. Visual acuity improved from an average of $6 / 24$ to $6 / 6$. Using the second technique, the average preoperative and postoperative ratings were 1.20 and 0.30 , respectively, with an average improvement of visual acuity from $6 / 36$ to $6 / 6$. There were no significant differences in any of the parameters evaluated between the first and the second techniques, and the degree of improvement in eyelid closure, keratopathy, and visual acuity was the same. No restriction of visual field was noted in either group. The mean preoperative palpebral fissure height was 11.7 (range 8-14) $\mathrm{mm}$ and the mean preoperative lagophthalmos was 8 (range 5-11) mm. Mean postoperative lagophthalmos was reduced to 2.6 (range 0-6) $\mathrm{mm}$ at 8 weeks following surgery. In three patients, a slight scleral show was present during forward gaze, which was assumed to be due to associated lower lid laxity.

No extrusion or infection was observed during the study, although seven patients who underwent the first technique had noticeable bulge in the upper eyelid and one patient had implant migration causing disfigurement of the upper eyelid. Implant migration was easily managed by repositioning of the gold plate under local anesthesia. Exploration of the previously placed gold implant was done with no further difficulty than the original procedure. Using the second technique, only three patients had noticeable bulge in the upper eyelid. Three patients had 
overcorrection (defined as ptosis $>2 \mathrm{~mm}$ ) after gold plate insertion using the first technique and only one using the second technique. Complications are shown in Table 2. The rate of ocular inflammation was 3-6 episodes per month, which reduced to $0-1$ per month using both techniques. One case of conjunctival perforation in one eye $(1.6 \%$ of all eyes) occurred using the second technique. Complete resolution of symptoms was observed in 24 eyes using the first technique $(63.1 \%)$, and in 15 eyes using the second technique $(65.2 \%)$.

\section{Discussion}

Patients with facial nerve palsy suffer from a range of eye problems, including eye dryness and repeated eye irritation and infection. ${ }^{11}$ These patients need eye protection using either a medical (eye lubricants, eye drops and occlusive dressings) or a surgical strategy. Gold plate placement can be used to treat lagophthalmos either temporarily until recovery of the paresis of the orbicularis muscle, or permanently if recovery does not occur. When there is improvement and complete recovery of the paresis, the gold weights can be removed. However, if there is partial recovery, the plates can be removed and replaced by smaller weights. ${ }^{12}$ Lower lid repositioning can also be done to treat lower lid laxity. Esthetic appearance is an important issue for patients, and the weights seem to mimic normal blinking of the eyes. ${ }^{13}$

In this study, the surgical techniques used to implant the gold plates, either at a high level or a low level, were the same, but the height from the lid margin was different. The standard technique in the literature is to place the gold plate $2-3 \mathrm{~mm}$ from the lid margin. In our early cases, we started by placing the gold plates at $2 \mathrm{~mm}$ from the lid margin, but in later cases, we tried to elevate the level to $5 \mathrm{~mm}$ from the lid margin, with the aim of hiding the plate behind the upper lid fold and decreasing the upper lid bulge.

A preoperative trial of placing gold weights on the outer surface is important to assess the weight of the gold plates intraoperatively. In our study, when placing the gold plate at a high level, heavier gold plates were needed to achieve the desired level of the lids. Fewer bulges were obvious in the upper eyelids. This gives a better esthetic look, but needs

Table 2 Complications occurring in both techniques

\begin{tabular}{lll}
\hline Complication & Group I (38 eyes) & Group 2 (23 eyes) \\
\hline Noticeable bulge & $7(18.4 \%)$ & $3(13 \%)$ \\
Migration of plate & $\mathrm{I}(2.6 \%)$ & - \\
Ptosis & $3(7.8 \%)$ & $\mathrm{I}(4.3 \%)$ \\
Conjunctival perforation & - & $\mathrm{I}(4.3 \%)$ \\
\hline
\end{tabular}

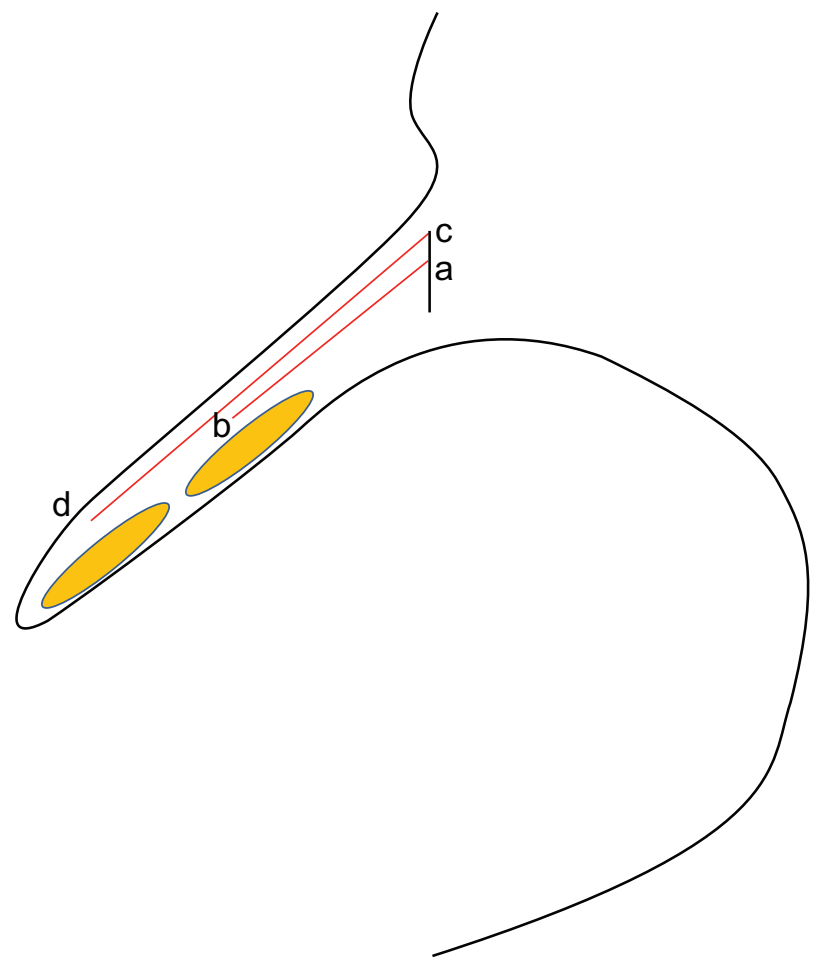

Figure 4 Showing $a b \times$ gold plate weight is less than $c d \times$ same gold plate weight.

a heavier weight to obtain lid closure. ${ }^{14}$ In eyes with lower level placement of the gold plates, the incidence of upper eyelid ptosis was higher, and there was a higher incidence of obvious upper eyelid bulge, which was annoying for the patients.

According to the mathematical equation of work $=$ force $\times S$, where in our study $\mathrm{S}$ represents the distance that can be moved by the gold plate on lid opening, and according to Figure 4, if we are using the same gold weight (force), we notice that the exerted work is more for the first technique, because $\mathrm{ab} \times$ gold plate weight is less than $\mathrm{cd} \times$ same gold plate weight. No significant differences existed for any of the evaluated parameters between the first and the second techniques, and the degree of improvement in eyelid closure, keratopathy, and visual acuity was the same. No restriction of visual field was noted in either group. Patients with high-level placement would need $0.2 \mathrm{~g}$ heavier gold plates than the lower-level placement to achieve sufficient load on the lids.

\section{Conclusion}

Placement of gold plates at a higher level could avoid some of the drawbacks of lower-level plate placement, including upper eyelid bulge and ptosis, especially given that there is thinning of the eyelids and orbicularis muscle in facial palsy. This study reports that a higher level of insertion of the gold plate achieves better esthetic results with less 
noticeable bulge in the upper eyelid and with acceptable functional results. However, we believe that a comparative study in a larger series is required to investigate further the most appropriate technique for gold plate insertion. Until then, case by case analysis and surgical decision-making is recommended.

\section{Disclosure}

The authors report no conflicts of interest in this work.

\section{References}

1. Beuerman RW, Schimmelpfennig B. Sensory denervation of the rabbit cornea affects epithelial properties. Exp Neurol. 1980;69:196-201.

2. Rubin LR, Lee GW, Simpson RL. Reanimation of the long-standing partial facial paralysis. Plast Reconstr Surg. 1986;77:41-49.

3. Sonmez A, Ozturk N, Durmus N, Bayramicli M, Numanoglu A. Patients' perspectives on the ocular symptoms of facial paralysis after gold weight implantation. J Plast Reconstr Aesthet Surg. 2008;61: 1065-1068.

4. Choo PH, Carter SR, Seiff SR. Upper eyelid gold weight implantation in the Asian patient with facial paralysis. Plast Reconstr Surg. 2000;105:855-859.

5. Oven EA. Lagophthalmos. In: Matayoshi S, Oven EA, Moura EM, editors Manual of Ocular Plastic Surgery. Sao Paulo, Brazil: Roca; 2004.
6. Portellinha WM, Genta CB, Sobrinho EFA, Cunha MC, Mura EM. Facial paralysis - lagophthalmos. In: Smith EJ, Moura EM, Goncalves OJ, editors. Ocular Surgery. Sao Paulo, Brazil: Roca; 1997.

7. Caesar RH, Friebel J, McNab AA. Upper lid loading with gold weights in paralytic lagophthalmos: a modified technique to maximize the long-term functional and cosmetic success. Orbit. 2004;23:27-32.

8. Kao CH, Moe KS. Retrograde weight implantation for correction of lagophthalmos. Laryngoscope. 2004;114:1570-1575.

9. Thomas DA, Khalifa YM. Temporalis fascia in the management of gold eyelid weight extrusion. Ophthal Plast Reconstr Surg. 2005;21: 153-155.

10. Bhatti AF, Page K, Orlando A. Modification of the gold weight implant for insertion into the upper eyelid in facial palsy. Ann Plast Surg. 2005;55: 689-692.

11. Seiffer SR, Sullivan JH, Freean LN, Ahn J. Pretarsal fixation of gold weights in facial nerve palsy. Ophthal Plast Reconstr Surg. 1989;5: 104-109.

12. Kinney SE, Seeley BM, Seeley MZ, Foster JA. Oculoplastic surgical techniques for protection of the eye in facial nerve paralysis. Am J Otol. 2000;21:275-280.

13. Sonmez A, Ozturk N, Durmus N, Bayramicli M, Numanoglu A. Patients' perspectives on the ocular symptoms of facial paralysis after gold weight implantation. J Plast Reconstr Aesthet Surg. 2008;61:1065-1068.

14. Kelly SA, Sharpe DT. Gold eyelid weights in patients with facial palsy: a patient review. Plast Reconstr Surg. 1992;89:436-440.
Clinical Ophthalmology

\section{Publish your work in this journal}

Clinical Ophthalmology is an international, peer-reviewed journal covering all subspecialties within ophthalmology. Key topics include: Optometry; Visual science; Pharmacology and drug therapy in eye diseases; Basic Sciences; Primary and Secondary eye care; Patien Safety and Quality of Care Improvements. This journal is indexed on

Submit your manuscript here: http://www.dovepress.com/clinical-ophthalmology-journal

\section{Dovepress}

PubMed Central and CAS, and is the official journal of The Society of Clinical Ophthalmology (SCO). The manuscript management system is completely online and includes a very quick and fair peer-review system, which is all easy to use. Visit http://www.dovepress.com/ testimonials.php to read real quotes from published authors. 